\title{
A retrospective clinical analysis of 5 cases of vaginal melanoma
}

\author{
REIKO TASAKA, TAKESHI FUKUDA, TAKUMA WADA, MASARU KAWANISHI, KENJI IMAI, \\ MARI KASAI, YASUNORI HASHIGUCHI, TOMOYUKI ICHIMURA, TOMOYO YASUI and TOSHIYUKI SUMI
}

Department of Obstetrics and Gynecology, Osaka City University Graduate School of Medicine, Osaka 545-8585, Japan

Received October 31, 2016; Accepted December 14, 2016

DOI: $10.3892 / \mathrm{mco} .2017 .1158$

\begin{abstract}
Vaginal melanoma is a rare tumor, accounting for $<1 \%$ of all melanomas and $\sim 1-5 \%$ of all vaginal malignant tumors. The prognosis of vaginal melanoma is extremely poor, as it is often resistant to chemotherapy and radiotherapy, and metastases may develop in the early stages of the disease. The present study investigated 5 patients with vaginal melanoma treated at the Department of Gynecology of Osaka City University Hospital (Osaka, Japan) between October, 2000 and April, 2014. All the cases presented with abnormal genital bleeding as the main complaint. Notably, in 3 of the 5 cases the tumors appeared as non-pigmented polyps. Local resection was performed as the primary treatment in all 5 cases. After surgery, dermal injection of interferon $\beta$ (feron maintenance therapy) was performed in 3 cases, and dacarbazine, nimustine, vincristine and interferon $\beta$ (DAVFeron therapy) was administered in 1 case as adjuvant therapy. All 5 cases recurred within 1 year. The site of recurrence varied, and included the vaginal wall, liver, brain and lung. The median overall survival was 419 days and the median progression-free survival 177 days. In this cohort, all the cases presented with abnormal genital bleeding as the main complaint. Therefore, malignant melanoma of the vagina must be considered along with other gynecological malignancies in patients with abnormal genital bleeding. In this study, over half of the cases had a non-pigmented polypoid lesion of the vagina. Therefore, malignant melanoma of the vagina must be considered when a polypoid lesion is identified on the vaginal wall.
\end{abstract}

\section{Introduction}

Vaginal melanoma is a rare tumor that accounts for $<1 \%$ of all melanomas and $~ 5 \%$ of all vaginal malignant tumors (1-4). The etiology of vaginal melanoma remains unknown at

Correspondence to: Dr Takeshi Fukuda, Department of Obstetrics and Gynecology, Osaka City University Graduate School of Medicine, 1-4-3 Asahimachi, Abeno-ku, Osaka 545-8585, Japan E-mail: takeshif@med.osaka-cu.ac.jp

Key words: vaginal melanoma, malignant melanoma, vaginal malignant tumor, vaginal polyp, abnormal genital bleeding present as, given its location, ultraviolet radiation is unlikely to be involved in the tumorigenic process.

The prognosis of vaginal melanoma is extremely poor, with a 5-year overall survival (OS) rate of only $\sim 18 \%$, which is significantly lower compared with that of vulvar melanoma (47\%) and cutaneous melanoma (81\%) (5). The occult nature of the anatomical location contributes to the late presentation and late diagnosis. In addition, the diffuse lymphatic vascular plexus in the vagina promotes early metastasis of vaginal melanoma.

There are currently no established guidelines for the treatment of vaginal melanoma due to its rarity; therefore, physicians may find deciding on the treatment method challenging. Complete resection may be difficult due to its anatomical location, and vaginal melanoma is often resistant to chemotherapy and radiotherapy (6). We herein present a retrospective clinical analysis of 5 cases of vaginal melanoma.

\section{Patients and methods}

Patients. A total of 5 patients with vaginal melanoma, treated at the Department of Gynecology of Osaka City University Hospital (Osaka, Japan) between October, 2000 and April, 2014, were investigated. This is a retrospective study of the patient characteristics and treatment outcomes. Ethics approval and written informed consent have been obtained. A staging system of vaginal melanoma has not been fully established to date; thus, the International Federation of Gynecology classification for vaginal cancer (7) was used to determine disease stage. In all 5 patients, the diagnosis of malignant melanoma was histologically confirmed, and staging was based on pelvic examination, computed tomography and magnetic resonance imaging.

The patients' medical records were consulted for information on patient characteristics, treatment, histological findings, presence of recurrence, site of recurrence, treatment for recurrence, OS and disease-free survival (DFS).

\section{Results}

Patient characteristics. The characteristics of the 5 cases are summarized in Tables I and II. All the cases presented with abnormal genital bleeding as the main complaint. Notably, in 3 of the 5 cases, the tumors appeared as non-pigmented polyps. In all 5 cases, local resection was performed as primary treatment. 
Table II. Patient characteristics $(\mathrm{n}=5)$.

\begin{tabular}{lc}
\hline Characteristics & No. (\%) \\
\hline Age, years [median (range)] & $78(62-82)$ \\
Main complaint & \\
Abnormal genital bleeding & $5(100)$ \\
Initial diagnosis & \\
Malignant melanoma & $2(40)$ \\
Vaginal polyp & $2(40)$ \\
Vaginal carcinoma & $1(20)$ \\
Stage & \\
IIa & $2(40)$ \\
IIc & $3(60)$ \\
Primary treatment & \\
Local resection & $5(100)$ \\
Adjuvant therapy & \\
Local injection of IFN- $\beta$ & $3(60)$ \\
DAVFeron & $1(20)$ \\
Not performed & $1(20)$
\end{tabular}

IFN- $\beta$, interferon $\beta$; DAVFeron, dacarbazine + nimustine + vincristine + IFN- $\beta$.

Treatment and recurrence. All 5 cases recurred within 1 year. The site of recurrence varied and included the vaginal wall, liver, brain and lung. Treatment for recurrence was as follows: An extended hysterectomy was performed in case 1, which recurred in the uterine cervix; X-Knife radiosurgery was used in case 3 for a brain metastasis; local resection was performed in case 5 for a recurrence in the vagina, and dacarbazine and nivolumab [an anti-programmed cell death protein-1 (PD-1) antibody] were administered to treat the second recurrence in the vagina and the multiple lung metastases; in case 2, nivolumab was administered for a liver metastasis; and in case 4, the patient declined additional treatment for the recurrence.

The survival curves for OS and DFS are illustrated in Figs. 1 and 2, respectively. The median OS was 419 days and the median DFS was 177 days.

The non-pigmented lesion of case 5 is presented in Fig. 3 . At the first visit, the possibility of melanoma was not taken into consideration, and a biopsy of the tumor suggested the diagnosis of squamous cell carcinoma. The histopathological examination following local resection established the diagnosis of malignant melanoma.

\section{Discussion}

The prognosis of vaginal melanoma is worse compared with that of cutaneous melanoma, vulvar melanoma, and other vaginal malignancies $(5,8-10)$. The occult nature of its anatomical location may contribute to the late presentation and late diagnosis of vaginal melanoma, and the diffuse lymphatic vascular plexus in the vagina contributes to the early metastasis of vaginal melanomas. 


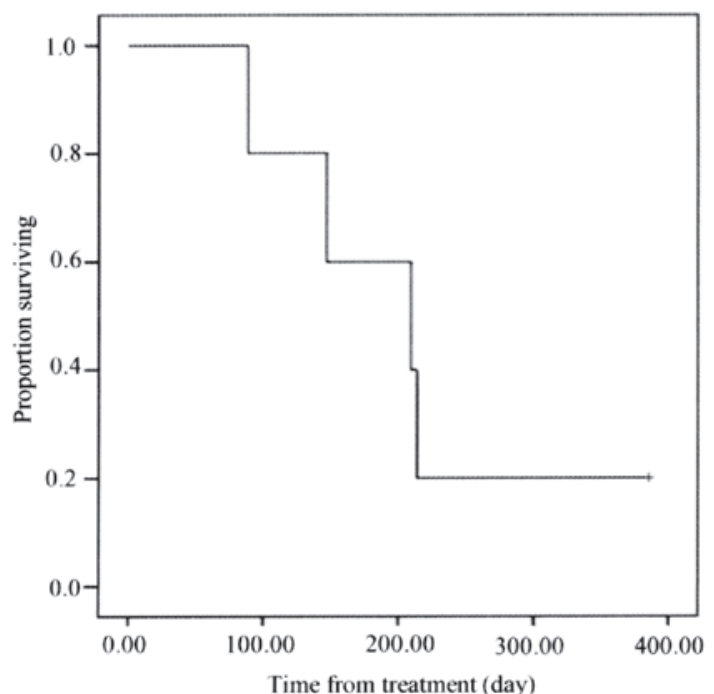

Figure 1. Overall survival (OS) curve. The median OS was 419 days.

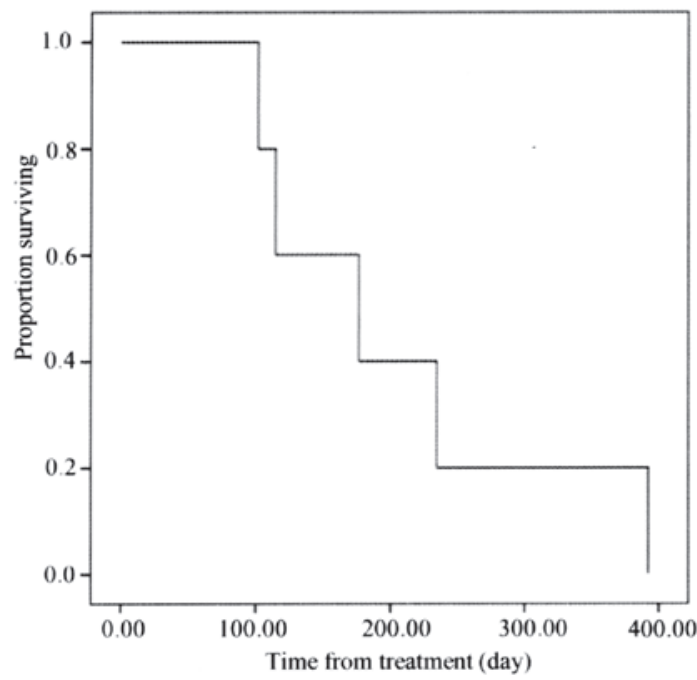

Figure 2. Disease-free survival (DFS) curve. The median DFS was 177 days.

The prognostic factors of vaginal melanoma have not been definitely determined. From the previous literature, tumor size, depth of invasion, lymph node status, extent of surgery and adjuvant therapy may affect the prognosis of vaginal melanoma (1).

Primary treatment protocols for vaginal melanoma remain to be established. Surgery, radiotherapy, chemotherapy and immunotherapy are recommended as individual and combined therapies. Surgery is the optimal treatment and is considered to be the only potentially curative treatment for vaginal melanoma (1). Surgical procedures for the primary disease range from conservative local excision to a more radical approach, including vaginectomy and pelvic exenteration. Since an association between the extent of surgery and survival has not been proven for vaginal melanoma, there has been significant controversy regarding the optimal surgical method. Therefore, local resection was performed in all the cases presented herein.

There is currently no standard approach to adjuvant therapy. The limited case series available suggest an
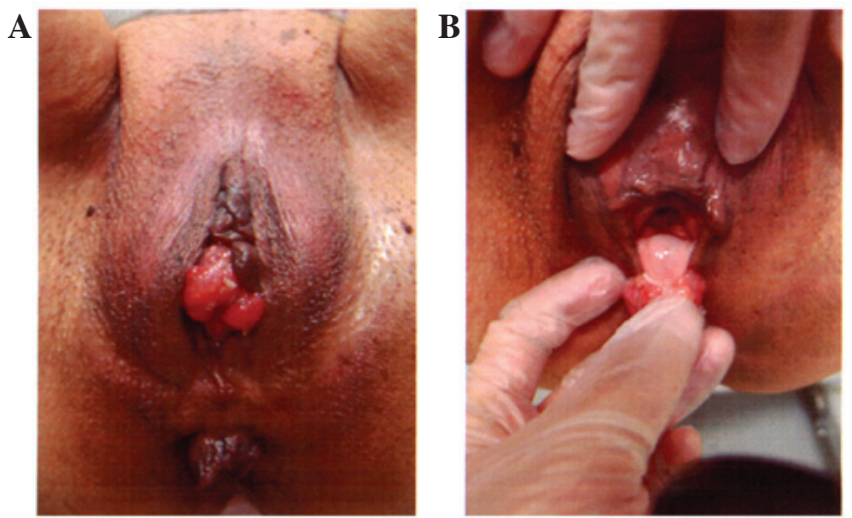

Figure 3. Case no. 5 exhibited a non-pigmented exophytic vaginal tumor with an irregular surface, which was confirmed by histopathological examination to be a malignant melanoma.

improvement in local control with the use of radiotherapy in the adjuvant setting for vulvar and vaginal melanomas. Additionally, for patients with limited treatment options, radiotherapy may also provide some palliative benefit in terms of symptomatic control (6). For advanced and recurrent disease, palliative systemic therapy may be an option. The authors of a retrospective case series of vulvar or vaginal lesions reported that, with treatment with a variety of biochemotherapy regimens (dacarbazine, cisplatin, interferon- $\alpha$ and interleukin-2), a partial response was achieved in $36 \%$ of the patients (11).

Systemic therapy for advanced cutaneous melanoma has changed significantly. There have been reports of significant OS improvement in response to agents such as anticytotoxic T-lymphocyte-associated antigen-4 antibodies (ipilimumab) $(12,13)$, antibodies against BRAF (vemurafenib and dabrafenib), MEK inhibitors (trametinib) (14-16) and an anti-PD-1 antibody (nivolumab) (17). Nivolumab may result in significant improvements in OS and progression-free survival as compared with dacarbazine for previously untreated patients with metastatic melanoma without a $B R A F$ mutation (17); thus, it may be helpful in the treatment of vaginal melanoma.

In the present study, all 5 cases presented with abnormal genital bleeding as the main complaint. Therefore, malignant melanoma of the vagina must be considered along with other gynecological malignancies in patients presenting with abnormal genital bleeding. In this study, 3 of the 5 cases had a non-pigmented polypoid lesion of the vagina. Therefore, the possibility of malignant melanoma of the vagina must be taken into consideration in patients with a polypoid lesion on the vaginal wall.

\section{References}

1. Xia L, Han D, Yang W, Li J, Chuang L and Wu X: Primary malignant melanoma of the vagina: A retrospective clinicopathologic study of 44 cases. Int J Gynecol Cancer 24: 149-155, 2014.

2. Bishop KD and Olszewski AJ: Epidemiology and survival outcomes of ocular and mucosal melanomas: A population-based analysis. Int J Cancer 134: 2961-2971, 2014.

3. Frumovitz M, Etchepareborda M, Sun CC, Soliman PT, Eifel PJ, Levenback CF and Ramirez PT: Primary malignant melanoma of the vagina. Obstet Gynecol 116: 1358-1365, 2010. 
4. Das P, Kumar N, Ahuja A, Jain A, Ray R, Sarkar C and Gupta SD Primary malignant melanoma at unusual sites: An institutional experience with review of literature. Melanoma Res 20: 233-239, 2010.

5. Ragnarsson-Olding B, Johansson H, Rutqvist LE and Ringborg U: Malignant melanoma of the vulva and vagina: Trends in incidence, age distribution, and long-term surviva among 245 consecutive cases in Sweden 1960-1984. Cancer 71: 1893-1897, 1993

6. Nobbenhuis MA, Lalondrelle S, Larkin J and Banerjee S: Management of melanomas of the gynaecological tract. Curr Opin Oncol 26: 508-513, 2014.

7. FIGO Committee on Gynecologic Oncology: Current FIGO staging for cancer of the vagina, fallopian tube, ovary, and gestational trophoblastic neoplasia. Int J Gynaecol Obstet 105: 3-4, 2009.

8. Creasman WT, Phillips JL and Menck HR: The National Cancer Data Base report on cancer of the vagina. Cancer 83: 1033-1040, 1998.

9. Piura B: Management of primary melanoma of the female urogenital tract. Lancet Oncol 9: 973-981, 2008.

10. Weinstock MA: Malignant melanoma of the vulva and vagina in the United States: Patterns of incidence and population-based estimates of survival. Am J Obstet Gynecol 171: 1225-1230, 1994.

11. Harting MS and Kim KB: Biochemotherapy in patients with advanced vulvovaginal mucosal melanoma. Melanoma Res 14: 517-520, 2004
12. Hodi FS, O'Day SJ, McDermott DF, Weber RW, Sosman JA, Haanen JB, Gonzalez R, Robert C, Schadendorf D, Hassel JC, et al: Improved survival with ipilimumab in patients with metastatic melanoma. N Engl J Med 363: 711-723, 2010.

13. Robert C, Thomas L, Bondarenko I, O'Day S, Weber J, Garbe C, Lebbe C, Baurain JF, Testori A, Grob JJ, et al: Ipilimumab plus dacarbazine for previously untreated metastatic melanoma. N Engl J Med 364: 2517-2526, 2011.

14. Hauschild A, Grob JJ, Demidov LV, Jouary T, Gutzmer R, Millward M, Rutkowski P, Blank CU, Miller WH Jr, Kaempgen E, et al: Dabrafenib in BRAF-mutated metastatic melanoma: A multicentre, open-label, phase 3 randomised controlled trial. Lancet 380: 358-365, 2012.

15. Chapman PB, Hauschild A, Robert C, Haanen JB, Ascierto P, Larkin J, Dummer R, Garbe C, Testori A, Maio M, et al: Improved survival with vemurafenib in melanoma with BRAF V600E mutation. N Engl J Med 364: 2507-2516, 2011.

16. Flaherty KT, Infante JR, Daud A, Gonzalez R, Kefford RF, Sosman J, Hamid O, Schuchter L, Cebon J, Ibrahim N, et al: Combined BRAF and MEK inhibition in melanoma with BRAF V600 mutations. N Engl J Med 367: 1694-1703, 2012.

17. Robert C, Long GV, Brady B, Dutriaux C, Maio M, Mortier L, Hassel JC, Rutkowski P, McNeil C, Kalinka-Warzocha E, et al: Nivolumab in previously untreated melanoma without BRAF mutation. N Engl J Med 372: 320-330, 2015. 